\title{
Progressive induction of left ventricular pressure overload in a large animal model elicits myocardial remodeling and a unique matrix signature
}

\author{
William M. Yarbrough, MD, ${ }^{\mathrm{a}}$ Rupak Mukherjee, PhD, ${ }^{\mathrm{b}}$ Robert E. Stroud, MS, ${ }^{\mathrm{b}}$ William T. Rivers, BS, ${ }^{\mathrm{b}}$ \\ J. Marshall Oelsen, BS, ${ }^{\mathrm{b}}$ Jennifer A. Dixon, MD, ${ }^{\mathrm{b}}$ Shaina R. Eckhouse, MD, ${ }^{\mathrm{b}}$ John S. Ikonomidis, MD, PhD, ${ }^{\mathrm{b}}$ \\ Michael R. Zile, MD, ${ }^{\mathrm{c}, \mathrm{d}}$ and Francis G. Spinale, MD, $\mathrm{PhD}^{\mathrm{b}, \mathrm{d}, \mathrm{e}}$
}

\begin{abstract}
Objective: Patients with severe left ventricular pressure overload secondary to aortic stenosis can present with signs and symptoms of heart failure despite normal left ventricular ejection fraction. This process occurs, at least in part, as a result of left ventricular pressure overload-induced extracellular matrix remodeling that promulgates increased left ventricular stiffness and impaired diastolic function. However, the determinants that drive extracellular matrix remodeling in this form of left ventricular pressure overload remain to be fully defined.
\end{abstract}

\begin{abstract}
Methods: Left ventricular pressure overload was induced in mature pigs $(n=15)$ by progressive ascending aortic cuff inflation (once per week for 4 weeks), whereby left ventricular mass, left ventricular ejection fraction, and regional myocardial stiffness $\left(\mathrm{rK}_{\mathrm{m}}\right)$ were compared with referent controls $(\mathrm{n}=12)$. Determinants of extracellular matrix remodeling were assessed by measuring levels of mRNA expression for fibrillar collagens, matrix metalloproteinases, and tissue inhibitors of matrix metalloproteinase 1 and 4.
\end{abstract}

\begin{abstract}
Results: With left ventricular pressure overload, left ventricular mass and $\mathrm{rK}_{\mathrm{m}}$ increased by 2- and 3-fold, respectively, compared with control, with no change in left ventricular ejection fraction. Left ventricular myocardial collagen increased approximately 2 -fold, which was accompanied by reduced solubility (ie, increased cross-linking) with left ventricular pressure overload, but mRNA expression for fibrillar collagen and matrix metalloproteinases remained relatively unchanged. In contrast, a robust increase in mRNA expression for tissue inhibitors of matrix metalloproteinase-1 and 4 occurred with left ventricular pressure overload.
\end{abstract}

Conclusions: In a progressive model of left ventricular pressure overload, which recapitulates the phenotype of aortic stenosis, increased extracellular matrix accumulation and subsequently increased myocardial stiffness were not due to increased fibrillar collagen expression but rather to determinants of post-translational control that included increased collagen stability (thereby resistant to matrix metalloproteinase degradation) and increased endogenous matrix metalloproteinase inhibition. Targeting these extracellular matrix posttranslational events with left ventricular pressure overload may hold both diagnostic and therapeutic relevance.

(J Thorac Cardiovasc Surg 2012;143:215-23)

Aortic stenosis gives rise to left ventricular (LV) pressure overload (LVPO). Without relief of LVPO, significant LV

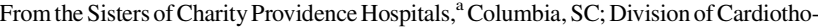
racic Surgery and Adult Cardiology, ${ }^{\text {b }}$ Medical University of South Carolina, ${ }^{\text {c }}$ Charleston, SC; Ralph H. Johnson Veterans' Administration Medical Center, ${ }^{\mathrm{d}}$ Charleston, SC; and University of South Carolina School of Medicine, ${ }^{\mathrm{e}}$ Columbia, SC. This work was supported by the Third Edward D. Churchill Research Scholarship (American Association for Thoracic Surgery) and a National Institutes of Health (NIH) Supplement award (HL 57952) to W.M.Y. Dr Eckhouse was supported by NIH Grant T32 HL007260. This project was also supported by NIH Grants HL057952, HL059165, and HL095608, and a Merit Award from the Veterans' Affairs Health Administration.

Disclosures: Authors have nothing to disclose with regard to commercial support.

Read at the 91st Annual Meeting of The American Association for Thoracic Surgery, Philadelphia, Pennsylvania, May 7-11, 2011.

Received for publication May 20, 2011; revisions received Sept 14, 2011; accepted for publication Sept 26, 2011; available ahead of print Nov 7, 2011.

Address for reprints: Francis G. Spinale, MD, PhD, Cardiovascular Translational

Research Center, CBA, University of South Carolina School of Medicine, 6439

Garners Ferry Road, Columbia, SC (E-mail: cvctrc@uscmed.sc.edu).

$0022-5223 / \$ 36.00$

Copyright (c) 2012 by The American Association for Thoracic Surgery

doi:10.1016/j.jtcvs.2011.09.032
}

hypertrophy occurs and is invariably associated with increased extracellular matrix (ECM) remodeling, most notably fibrillar collagen accumulation. ${ }^{1-4}$ LVPO with ECM remodeling can cause increased LV myocardial stiffness, impaired diastolic function, and signs and symptoms of heart failure (ie, diastolic dysfunction), despite relatively preserved LV systolic function, such as normal LV ejection fractions. ${ }^{1-4}$ Furthermore, clinical observations suggest the ECM remodeling that occurs with LVPO secondary to aortic stenosis is not readily reversible, despite a complete removal of the overload stimulus. ${ }^{1-4}$ Moreover, these persistent changes within the myocardial ECM have been associated with significant alterations in physiologic and clinical outcomes, such as LV myocardial stiffness and survival. ${ }^{5}$ Thus, identifying the specific mechanisms by which ECM remodeling occurs in the relevant context of LVPO holds both scientific and clinical relevance. Although a large number of studies pertaining to LVPO have been performed in rodents, most notably mice, these model systems 


\author{
Abbreviations and Acronyms \\ $\mathrm{ECM}=$ extracellular matrix \\ $\mathrm{LV}=$ left ventricular \\ $\mathrm{LVPO}=$ left ventricular pressure overload \\ MMP $=$ matrix metalloproteinase \\ TIMP $=$ tissue inhibitor of matrix \\ metalloproteinase
}

typically consist of an abrupt and acute induction of the pressure overload stimulus. ${ }^{6,7}$ As a result, in these murine models of acute LVPO induction, LV systolic function invariably decreases early and precipitously, which may not necessarily recapitulate the clinical context of LVPO. Large animal models of progressive LVPO have been described, whereby sequential induction of the pressure overload stimulus was performed, and thereby provides more relevant changes in LV structure and function to that of the clinical phenotype of aortic stenosis. ${ }^{8-11}$ Accordingly, the overall goal of this project was to develop a large animal model of LVPO that recapitulates the clinical phenotype of aortic stenosis and then examine potential transcriptional and post-transcriptional pathways that may contribute to the changes in myocardial ECM remodeling in this process.

The myocardial ECM is a complex entity that contains structural proteins, such as fibrillar collagens, nonstructural proteins, signaling molecules, and an array of proteases. ${ }^{12,13}$ In light of the fact that past studies have identified that the fibrillar collagens can influence LV myocardial stiffness properties in the context of LVPO, this was the initial focus of the present study. ${ }^{1-4}$ In terms of the fibrillar collagen matrix, an orchestrated set of events occurs with respect to expression, synthesis, cross-linking, and degradation/turnover. ${ }^{14,15}$ Accordingly, the first objective of the present study was to measure fibrillar collagen expression, overall content, and indices of collagen cross-linking in this large animal model of LVPO. A family of proteases that play a critical role in ECM degradation are the matrix metalloproteinases (MMPs), whereby the subclasses of these MMPs demonstrate different substrate specificities and biological function. ${ }^{12}$ Thus, the second objective of the present study was to measure the expression of representative MMPs from each subclass in this model of LVPO. A control point for overall MMP proteolytic activity is through endogenous MMP inhibition (tissue inhibitor of matrix metalloproteinase [TIMP]). ${ }^{12}$ Therefore, the third objective of the present study was to quantify targeted TIMP expression with LVPO. The central hypothesis of this study was that in this large animal model of LVPO, increased LV regional myocardial stiffness would occur in direct association with fibrillar collagen accumulation and specific posttranscriptional events in fibrillar collagen processing.

\section{MATERIALS AND METHODS}

The present study developed a model of progressive LVPO in mature pigs with the overarching aim of inducing significant LV hypertrophy without a compromise on LV ejection fraction, thereby simulating the clinical phenotype of LVPO. After the development of significant LV hypertrophy, the present study performed a set of integrated in vivo and ex vivo studies to define the relationship between changes in regional LV myocardial stiffness and that of myocardial collagen content and stability and MMP/ TIMP profiles. All animals were treated and cared for in accordance with the National Institutes of Health "Guide for the Care and Use of Laboratory Animals" (National Research Council, Washington, DC, 1996).

\section{Model of Progressive Left Ventricular Pressure Overload}

Mature Yorkshire pigs ( $\mathrm{n}=15,15 \pm 1 \mathrm{~kg}$; Hambone Farms, Orangeburg, SC) were anesthetized with isoflurane $(3 \% / 1.5 \mathrm{~L} / \mathrm{min})$ and nitrous oxide $(0.5 \mathrm{~L} / \mathrm{min})$. Through a left thoracotomy, a $6 \mathrm{~F}$ vascular access catheter (SlimPort M.R.I. Ultra Low Profile Implanted Port, Bard Access Systems, Salt Lake City, UT) was inserted and fixed within the descending thoracic aorta, and its port attachment was positioned within a subcutaneous pocket. The pericardium was incised, and a size-matched 12-mm inflatable Silastic vascular cuff (In Vivo Metric Biomedical Products, Healdsburg, Calif) was secured around the supracoronary ascending aorta without inducing aortic constriction. A uniform length of Silastic tubing was connected to a second subcutaneous access port for subsequent and serial engagement (Figure 1). The animals were allowed to recover for 1 week.

\section{Experimental Design}

At baseline (day 0 ) and at weekly intervals thereafter $(\times 4)$, the pigs were sedated with diazepam ( $200 \mathrm{mg}$ orally; Barr Laboratories, Pomona, NY) and the aortic lines were accessed in sterile fashion for plasma collection (Figure 1). Transthoracic echocardiographic images (ATL Ultramark VI, 2.25 MHz transducer, Bothell, Wash) of LV mass, volumes, and ejection fraction were obtained as described previously, ${ }^{16,17}$ and pressure gradients were computed from echocardiographic-generated Doppler velocities. On days $0,7,14$, and 21 , the access ports for the vascular occluders were engaged and injected with fixed and previously determined volumes of a $10 \%$ glycerol solution (in phosphate-buffered saline) to induce controlled and progressive LVPO. At 5 weeks post-LVPO, terminal measurements were performed to measure indices of LV myocardial stiffness and for tissue collection. Before the terminal end point study, decanted plasma was subjected to TIMP-1 and 4 analysis using a 2-laser flow cytometric detection system (Bio-Plex 200, BioRad Laboratories, Hercules, Calif) and a previously validated TIMP platform (TIMP MSA, LKT003; R\&D Systems, Minneapolis, Minn). ${ }^{17}$ By using precalibrated standards and regression modeling, the fluorescence emission was converted to an absolute TIMP concentration.

\section{Terminal Studies}

After sedation with diazepam (200 mg orally), anesthesia was induced with sufentanil ( $2 \mu \mathrm{g} / \mathrm{kg}$ intravenously; Baxter Healthcare Corp, Deerfield, Ill) and etomidate $(0.3 \mathrm{mg} / \mathrm{kg}$ intravenously, Bedford Laboratories). After endotracheal intubation, mechanical ventilation was initiated, and a stable anesthetic plane was achieved with morphine sulfate $(3 \mathrm{mg} / \mathrm{kg} / \mathrm{h}$ intravenously; Elkins-Sinn, Cherry Hill, NJ) and isoflurane $\left(1 \%, 3 \mathrm{~L} / \mathrm{min} \mathrm{O}_{2}\right.$; Baxter Healthcare Corp). A median sternotomy was performed, the pericardial sac was opened, and a pair of piezoelectric crystals $(2 \mathrm{~mm}$; Sonometrics, London, Ontario, Canada) was positioned against the LV anterior free wall endocardial surface to measure segmental wall motion. ${ }^{16}$ A previously calibrated microtipped transducer catheter was placed in the LV via a small apical incision (7.5F; Millar Instruments Inc, Houston, Tex). A vessel loop was placed around the inferior vena cava for transient caval occlusions. Pressure waveforms and crystal signals were digitized on a computer for subsequent analysis at a sampling frequency of $1 \mathrm{kHz}$ under 


\section{Transthoracic Echocardiography I Plasma Collection}
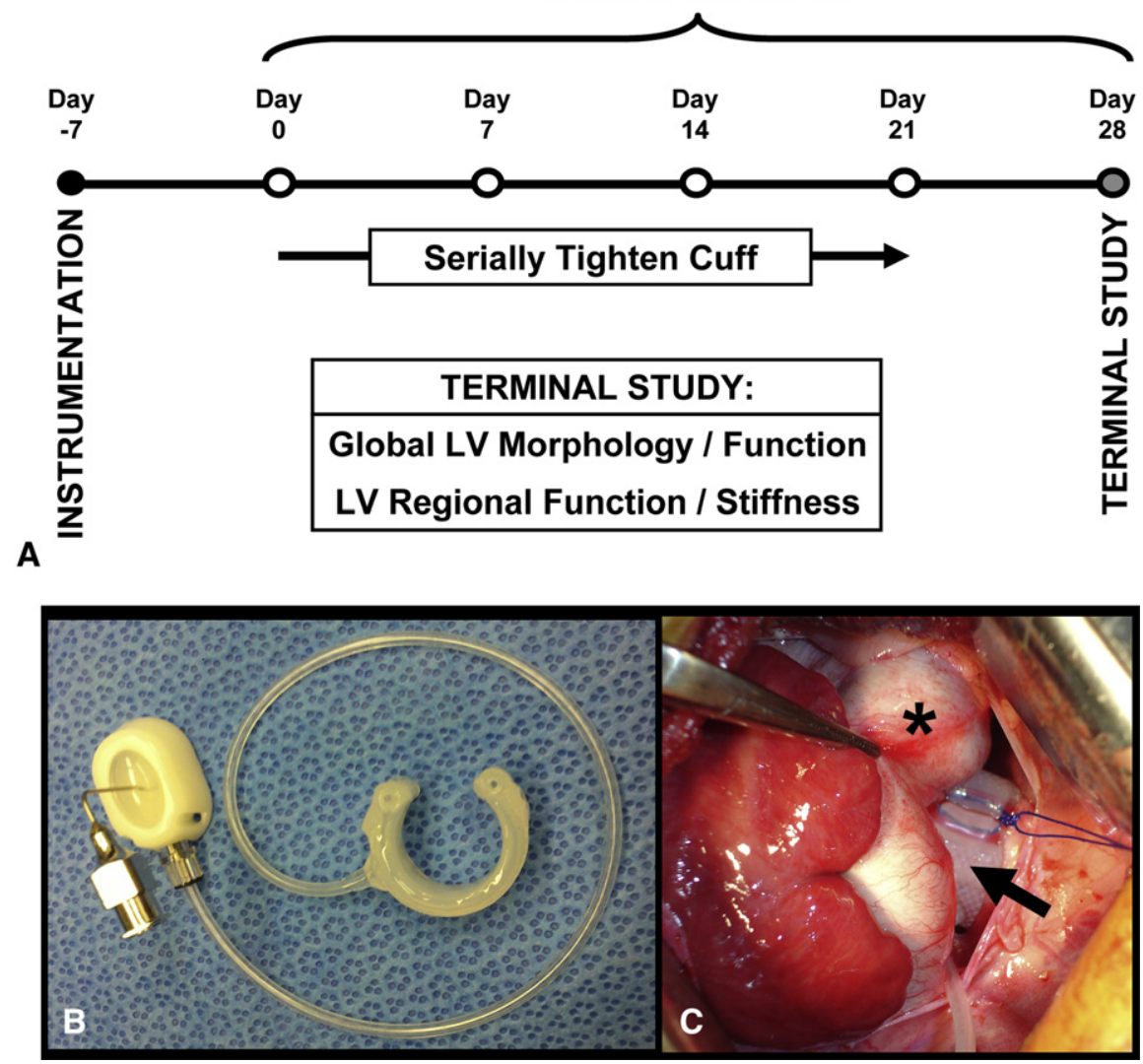

FIGURE 1. A, Experimental design: progressive cuff inflation was used to recapitulate the LV phenotype of LVPO secondary to AS. B, Disengaged 12-mm inflatable Silastic cuff connected to an implantable subcutaneous port accessed with an angled Huber needle. C, Intraoperative photograph demonstrates retraction of the main pulmonary artery $\left(^{*}\right)$ with fixation of a 12-mm inflatable Silastic cuff around the supracoronary ascending aorta (arrow). $L V$, Left ventricular.

steady-state conditions and during occlusion and release of the caval occlusion, and thereby constructing a family of LV pressure-dimension curves. The regional myocardial stiffness constant $\left(\mathrm{rK}_{\mathrm{m}}\right)$ was computed using the logarithmic transformation of the exponential end-diastolic stress-strain relationship as follows: $\sigma=\mathrm{A} \mathrm{e}^{(\mathbf{r K m} \varepsilon)}+\mathrm{B}$, where $\sigma$ is regional enddiastolic wall stress, $\mathrm{A}$ and $\mathrm{B}$ are constants, and $\varepsilon$ is the regional strain. ${ }^{17}$ The hearts were extirpated, and the LV posterior free wall, which was devoid of any instrumentation, was sectioned and snap-frozen in liquid nitrogen for subsequent biochemical analysis of collagen content and MMP and TIMP expression. ${ }^{18}$ An additional full-thickness section $(2 \times 2 \mathrm{~cm})$ was immersed in $4 \%$ formalin solution and processed for histomorphometric methods. Twelve normal, age-matched, non-instrumented pigs were included for comparison purposes.

\section{RNA Isolation and Real-Time Polymerase Chain Reaction}

LV samples ( $\sim 1 \mathrm{~g}$ ) were homogenized via high-speed shaking (RNeasy Fibrous Tissue Mini Kit, Qiagen, Valencia, Calif), and total RNA was extracted in the presence of DNase. Purified total RNA ( $1 \mathrm{~g})$ was immediately reverse transcribed to generate cDNA (iScript cDNA Synthesis Kit; BioRad Laboratories). All cDNA was stored at $-80^{\circ} \mathrm{C}$ until analyzed by quantitative polymerase chain reaction. The cDNA was amplified with gene-specific primer/probe sets (Universal PCR Master Mix with no AmperErase UNG;Cat\# 4364321, Applied Biosystems, Foster City, Calif).
The specific TaqMan primer/probe sets (Applied Biosystems) were MMP-2 (Cat\# Ss03394318_m1), MMP-9 (Cat\# Ss03392098_m1), MMP-7 (Cat\# Ss03383345_u1), MMP-13 (Cat\# Ss03373279_m1), MT1MMP (Cat\# Ss03394431_m1), TIMP-1 (Cat\# Ss03381944_u1), TIMP-4 (Cat\# Hs00162784_m1), collagen 1A1 (Cat\# Ss03373340_m1), and collagen 3A1 (Cat\# Ss03375691_g1). In addition, the internal control primer/probe sets for glucuronidase beta (Gusb, Applied Biosystems, Cat\# Ss03387751_u1 and 18s rRNA, Applied Biosystems, Cat\# Hs99999901_s1, eukaryotic $18 \mathrm{~S}$ ribosomal rRNA) were analyzed to assess constant expression levels across samples. Negative controls were run to verify the absence of genomic DNA contamination (reverse transcription control) and the absence of overall DNA contamination in the polymerase chain reaction system and working environment (template control). The real-time polymerase chain reaction fluorescence signal was converted to cycle times and normalized to the mean of the Gusb and 18-s signals.

\section{Collagen Content and Solubility}

LV myocardial samples were sequentially extracted as previously described. ${ }^{19}$ Briefly, samples were homogenized in extraction/homogenization buffer ( $10 \mathrm{mmol} / \mathrm{L}$ cacodylic acid pH 5.0, 0.15 mol/L NaCl, $20 \mathrm{mmol} / \mathrm{L} \mathrm{ZnCl}$, $1.5 \mathrm{mmol} / \mathrm{L} \mathrm{NaN}_{3}$, and $0.01 \%$ Triton X-100 [v/v]) and centrifuged $(800 g, 10$ minutes, $4^{\circ} \mathrm{C}$ ). The supernatant was removed containing soluble collagen, and the remaining pellet contained insoluble collagen. Protein concentrations for both fractions were determined (Bicinchoninic Acid protein assay, 23225; 

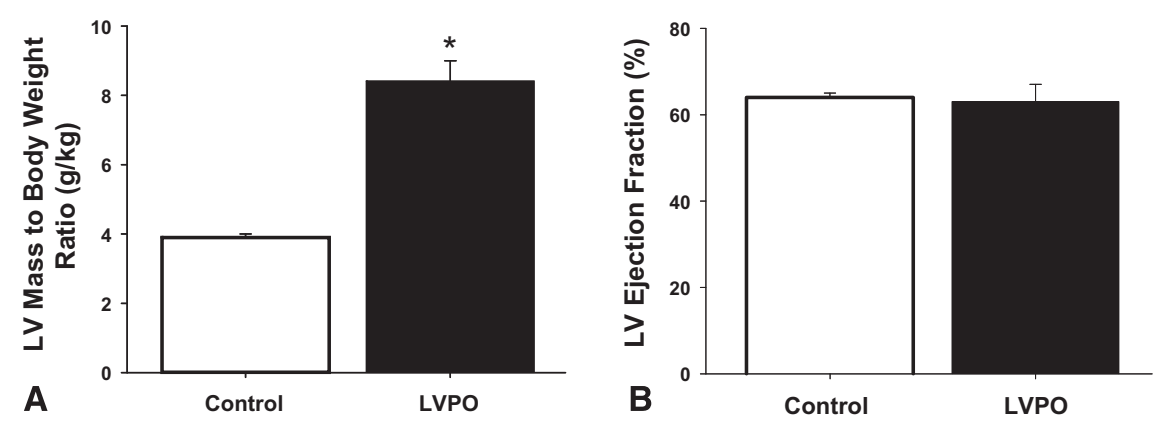

FIGURE 2. A, LVPO was associated with a marked increase in the LV mass to body weight ratio. B, The LV ejection fraction remained unchanged. Thus, this gradual induction of LVPO caused significant $L V$ hypertrophy without a compromise in $L V$ ejection fraction. $* P<.05$ vs control. $L V$, Left ventricular; $L V P O$, left ventricular pressure overload.

Thermo Scientific, Rockford, Ill). Collagen concentrations were then determined using a spectrophotometric assay with picrosirius red. ${ }^{19-21}$ Briefly, $60 \mu \mathrm{g}$ of collagen extract and a range of collagen standards (1-64 $\mu \mathrm{g}$; Collagen Type I, Cat\# 08-115, Millipore, Temecula, Calif), were placed in a 96-well microtiter plate, dried overnight, washed, and incubated with $0.1 \%$ Sirius red F3BA in picric acid (w/v; 1 hour), destained briefly with $10 \mathrm{mmol} / \mathrm{L} \mathrm{HCl}$, and washed. The plate was then subjected to spectrophotometry (540 nm; VersaMax, Molecular Devices, Sunnyvale, Calif). The soluble, insoluble, and thus total collagen content per nanogram of total protein was determined from the standard curve, and the collagen levels were divided by the original tissue weight to obtain soluble, insoluble, and total collagen per gram of myocardium.

\section{Left Ventricular Myocyte Cross-Sectional Area}

For the histomorphometry studies, sections $(5 \mu \mathrm{m})$ were stained with hematoxylin-eosin and imaged at a final magnification of $20 \times$. Ten random fields within the mid-myocardial region, devoid of any vascular compartment, were digitized, and myocyte cross-sectional area was computed (Sigma Scan, Media Cybernetics Inc, Bethesda, Md). Only those myocyte profiles whereby the nucleus was centrally located were included in this analysis. ${ }^{18}$

\section{Data Analysis}

Comparisons between the control and LVPO groups were performed using the Student $t$ test. Linear regression analysis was performed to determine correlations between indices of LV diastolic function, such as regional myocardial stiffness $\left(\mathrm{rK}_{\mathrm{m}}\right)$ to that of collagen biochemistry and myocyte hypertrophy. Statistical analyses were performed using the STATA package (Version 8, Intercooled; StataCorp, College Station, Tex). Data are presented as mean \pm standard error of the mean.

\section{RESULTS}

\section{Left Ventricular Function and Geometry}

In this model of gradual LVPO, the LV mass to body weight ratio doubled and the LV ejection fraction remained unchanged in the setting of LVPO when compared with referent age-matched controls (Figure 2). The effects of LVPO on LV geometry and function are summarized in Table 1. Although LV mass increased in the LVPO group, body weight was similar to that of referent controls. LVPO resulted in a peak terminal transaortic gradient of $66 \pm 6$ $\mathrm{mm} \mathrm{Hg}$ without hemodynamic compromise as evidenced by stability with respect to heart rate, mean arterial pressure, and cardiac output. In addition, LV end-diastolic volume remained unchanged from referent control values. However, in marked contrast, indices of LV diastolic function were significantly impaired in this model of LVPO, as evidenced by elevated end-diastolic pressure and reduced active relaxation rates (tau, negative $\mathrm{dP} / \mathrm{dt}$ ). Moreover, a 3-fold increase in regional myocardial stiffness $\left(\mathrm{rK}_{\mathrm{m}}\right)$ was observed with LVPO. Thus, this large animal model of LVPO resulted in significant LV hypertrophy with no significant compromise on LV systolic function, but significantly impaired diastolic function.

TABLE 1. Left ventricular geometry and function with chronic pressure overload

\begin{tabular}{|c|c|c|}
\hline & Control & LVPO $\ddagger$ \\
\hline Heart rate (beats/min) & $106 \pm 5$ & $114 \pm 10$ \\
\hline Mean arterial pressure $(\mathrm{mm} \mathrm{Hg})$ & $86 \pm 3$ & $92 \pm 5$ \\
\hline Cardiac output $(\mathrm{L} / \mathrm{min}) \dagger$ & $3.5 \pm 0.3$ & $3.3 \pm 0.3$ \\
\hline Pressure gradient $(\mathrm{mm} \mathrm{Hg}) \dagger$ & $1 \pm 1$ & $66 \pm 6^{*}$ \\
\hline Body weight (kg) & $31.6 \pm 1.2$ & $28.4 \pm 1.2$ \\
\hline LV end-diastolic volume $(\mathrm{mL}) \dagger$ & $50 \pm 2$ & $51 \pm 3$ \\
\hline \multicolumn{3}{|l|}{ LV systolic function } \\
\hline LV peak pressure $(\mathrm{mm} \mathrm{Hg})$ & $100 \pm 3$ & $182 \pm 8^{*}$ \\
\hline Peak positive $\mathrm{dP} / \mathrm{dt}(\mathrm{mm} \mathrm{Hg} / \mathrm{s})$ & $1601 \pm 66$ & $2861 \pm 272 *$ \\
\hline $\begin{array}{l}\text { Peak positive } \mathrm{dP} / \mathrm{dt} \text { normalized to } \\
\mathrm{LV} \text { peak pressure }\left(\mathrm{s}^{-1}\right)\end{array}$ & $16.3 \pm 1.0$ & $16.0 \pm 1.6$ \\
\hline \multicolumn{3}{|l|}{ LV diastolic function } \\
\hline LV end-diastolic pressure $(\mathrm{mm} \mathrm{Hg})$ & $8 \pm 1$ & $18 \pm 1^{*}$ \\
\hline Peak negative $\mathrm{dP} / \mathrm{dt}(\mathrm{mm} \mathrm{Hg} / \mathrm{s})$ & $2269 \pm 206$ & $3186 \pm 164^{*}$ \\
\hline $\begin{array}{l}\text { Peak negative } \mathrm{dP} / \mathrm{dt} \text { normalized to } \\
\mathrm{LV} \text { peak pressure }\left(\mathrm{s}^{-1}\right)\end{array}$ & $22.8 \pm 1.9$ & $17.7 \pm 0.9 *$ \\
\hline Tau (ms) & $29 \pm 1$ & $37 \pm 1^{*}$ \\
\hline Regional myocardial stiffness (rKm) & $3.9 \pm 0.3$ & $11.2 \pm 0.6^{*}$ \\
\hline \multicolumn{3}{|l|}{ Plasma TIMP profiles } \\
\hline TIMP-1 (pg/mL) & $44.7 \pm 8.0$ & $135.8 \pm 17.5^{*}$ \\
\hline TIMP-4 (pg/mL) & $74.6 \pm 9.5$ & $129.4 \pm 14.9^{*}$ \\
\hline Sample size (n) & 12 & 15 \\
\hline
\end{tabular}



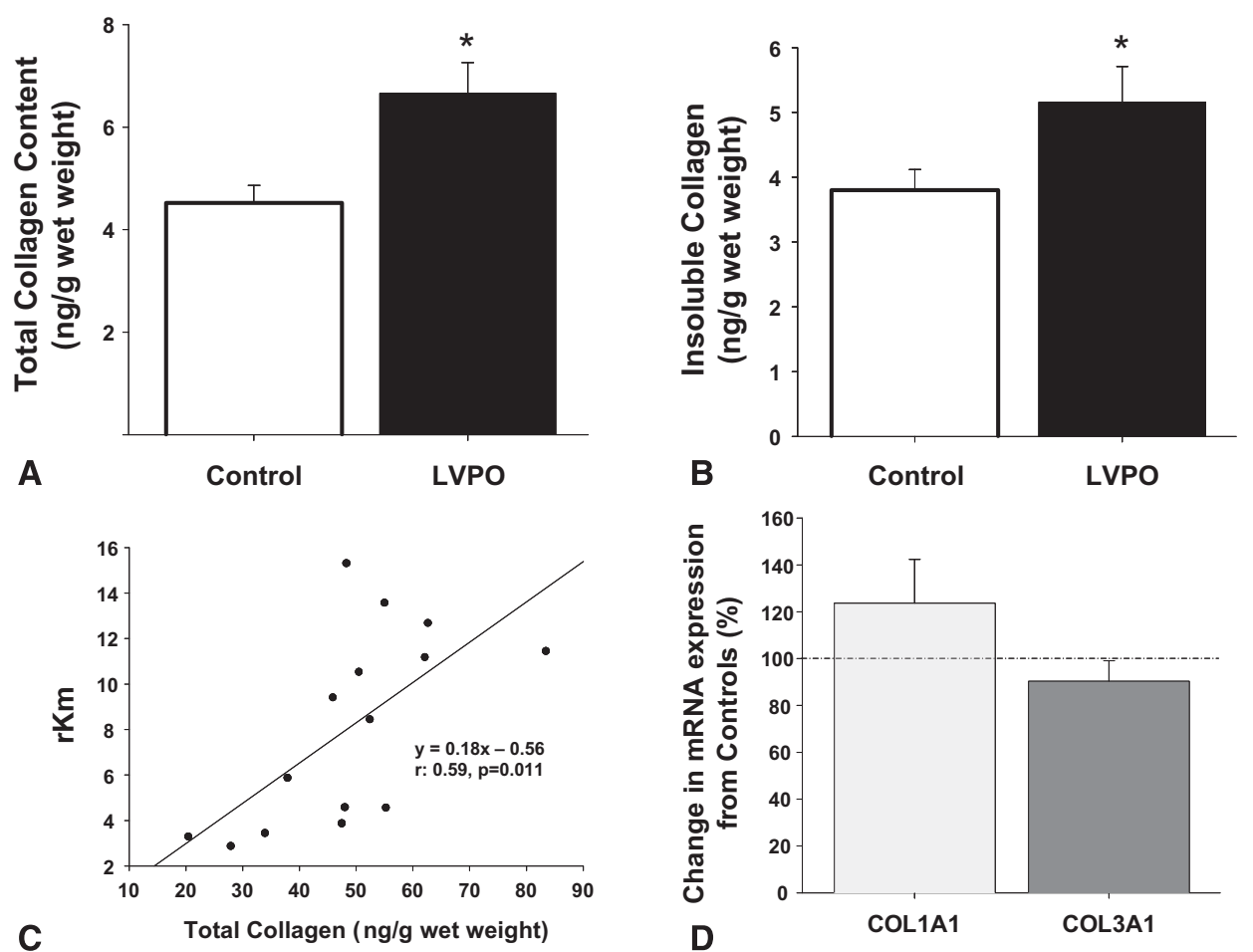

FIGURE 3. A, Total collagen content was greater with LVPO. B, Total collagen content is determined by soluble and insoluble collagen components. The soluble:insoluble collagen ratio changed with LVPO and favored an increase in insoluble collagen content indicating increased collagen cross-linking and collagen stability. C, A relationship between myocardial collagen content and regional myocardial stiffness was evident. D, Collagen 1A1 and collagen 3A1 mRNA expression did not change with LVPO. Thus, the observed increase in collagen content was not due to an increase in fibrillar collagen expression. $L V P O$, Left ventricular pressure overload. $* P<.05$ versus control.

\section{Left Ventricular Myocyte Hypertrophy, Collagen Content, and Expression}

By using histomorphometric methods, LV myocyte cross-sectional area increased by approximately 2 -fold with LVPO when compared with referent controls $(424+$ $22 \mu \mathrm{m}^{2}$ vs $273+5 \mu \mathrm{m}^{2}$, respectively, $\left.P<.05\right)$. Changes in total myocardial collagen content and solubility with LVPO are summarized in Figure 3. Total myocardial collagen content increased by approximately $50 \%$ with LVPO and was associated with a greater insoluble collagen fraction- indicative of reduced solubility. However, although total myocardial collagen increased, there was no significant change in relative fibrillar collagen type I or type III mRNA levels (Figure 3). Thus, this model of LVPO resulted in significant collagen accumulation and decreased solubility, but no change in the relative expression of the most common fibrillar collagen types.

\section{Left Ventricular Myocardial Matrix}

Metalloproteinase/Tissue Inhibitors of Matrix

Metalloproteinase mRNA Levels and Tissue

Inhibitors of Matrix Metalloproteinase Plasma Profiles

The relative levels for the different classes of MMP types and for TIMP-1 and TIMP-4 with respect to referent control values are summarized in Figure 4. Relative mRNA levels for MMPs and TIMPs are summarized in Figure 4. No change in mRNA levels was observed with LVPO for the interstitial collagenase (MMP-13) or gelatinase MMP subclasses. However, in the setting of LVPO, mRNA levels for matrilysin (MMP-7) decreased and mRNA levels for the membrane type MMP (MT1-MMP) increased. A robust increase in mRNA expression for TIMP-1 and 4 was observed with LVPO. The significantly increased myocardial TIMP-1 and 4 mRNA levels were paralleled by a 3-fold increase in plasma TIMP- 1 and more than a $50 \%$ increase in plasma TIMP-4 (Table 1).

\section{Relationship Among Left Ventricular Function, Hypertrophy, and Collagen}

A correlation matrix was developed to determine the relative association between indices of LV function, particularly diastolic function, to biochemical and morphometric indices of myocardial remodeling with LVPO. As would be expected, LV mass was strongly associated with both myocyte cross-sectional area and total collagen content ( $r=0.80$ and 0.67 , respectively, both $P<.05$ ). LV isovolumic relaxation and end-diastolic pressure were both associated with myocyte cross-sectional area $(r=0.60$ and 0.75 , respectively). For LV myocardial collagen 


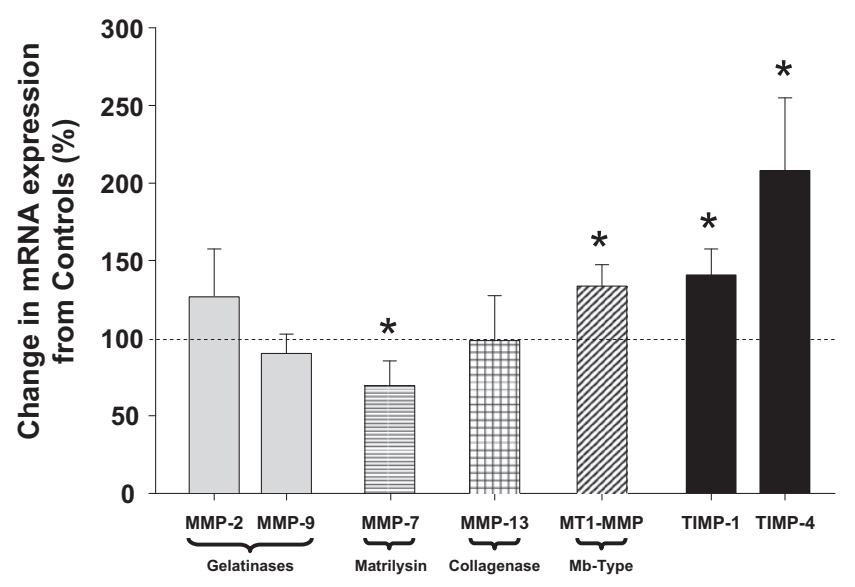

FIGURE 4. Determinants of ECM remodeling and fibrosis in the setting of LVPO include the MMPs and TIMPs. The overall MMP:TIMP ratio favored reduced collagen degradation with LVPO. $M M P$, Matrix metalloproteinase; TIMP, tissue inhibitor of matrix metalloproteinase. $* P<.05$ versus control.

content, one of the strongest relationships was between myocardial collagen content and regional myocardial stiffness (Figure 3).

\section{DISCUSSION}

Although LVPO is a common clinical problem caused by both hypertension and aortic stenosis, animal models that recapitulate the clinical LV phenotype have not been frequently used. LV functional and structural manifestations of LVPO include impaired LV filling (ie, diastolic dysfunction) without an early compromise in systolic function and LV hypertrophy with myocardial fibrillar collagen accumulation. Although a large number of studies have induced LVPO in murine models ${ }^{6,7}$ and provided invaluable molecular clues as to the adaptation of the LV to pressure overload stimuli, these murine constructs may not accurately reflect the clinical LV phenotype of LVPO. Accordingly, the present study used a large animal of LVPO to identify specific mechanisms and pathways that may contribute to ECM remodeling with LVPO, and thereby contribute to the LV myocardial stiffness that invariably occurs in this pathologic condition. The new and unique findings from the present study were 2-fold. First, gradual induction of LVPO in a large animal model resulted in LV hypertrophy and increased myocardial stiffness, and increased collagen matrix accumulation without a reduction in $\mathrm{LV}$ ejection fraction, thus recapitulating important features of the clinical phenotype of LVPO. Second, the collagen accumulation observed in this model of LVPO was not associated with a robust increase in collagen expression at the transcriptional level, but rather with major changes in post-translational processes, such as increased collagen cross-linking and TIMP induction. These new findings challenge the canonical concept that the increased collagen accumulation with LVPO is simply due to enhanced collagen expression and synthesis, but rather due to shifts in post-translational processes such as resistance to degradation and reduced proteolysis.

\section{Left Ventricular Pressure Overload and the Myocardial Extracellular Matrix}

Relief of LVPO, as is typically achieved by surgical correction of aortic stenosis, facilitates regression of LV hypertrophy. ${ }^{1,22}$ However, structural abnormalities persist within the myocardial ECM despite appropriate elimination of the LVPO stimulus and can be associated with suboptimal clinical outcomes. ${ }^{5,23}$ For example, Villari and colleagues ${ }^{2}$ demonstrated that increased ECM accumulation persists up to several years after aortic valve replacement when performed for long-standing LVPO. In addition, a recent investigation conducted by Azevedo and colleagues ${ }^{5}$ demonstrated that the magnitude of myocardial matrix accumulation at the time of aortic valve surgery was directly related to postoperative survival. These observations suggest that in patients with LVPO, an irreversible shift in ECM remodeling occurs, after which complete normalization of LV matrix structure may be precluded. The present study began to address this clinically relevant issue through identifying contributory mechanisms that likely underlie ECM remodeling with LVPO. The outcomes from this initial investigation suggest that specific post-translational pathways are altered in this pathologic process. Moreover, this LVPO model may be useful in guiding the identification of specific and potentially irreversible steps in the ECM remodeling in the context of LVPO. However, it must be recognized that a major determinant of the myocardial remodeling process with LVPO is predominantly characterized by myocyte hypertrophy, which was demonstrated in the present study. Indeed, the present study demonstrated strong associations between the indices of active relaxation and the degree of myocyte hypertrophy. Thus, it is likely the summation of both cellular and extracellular processes that give rise to significantly impaired LV filling and ultimately the presentation of heart failure. Another important consideration of the present study is that only indices of fibrillar collagen content and expression were considered, and these structural components actually comprise a small volume fraction of the entire ECM. The present study did not consider other constituents of the myocardial ECM, which include membrane bound and soluble bioactive molecules, as well as other proteins that contribute to the structure and biophysical properties of the ECM, such as the proteoglycans and glycosaminoglycans. Nevertheless, the present study does provide a foundation for future studies in a relevant large animal model of LVPO by which to examine other constituents of the ECM and critical signaling molecules that likely 
contribute to the myocardial remodeling process with this pathologic stimulus.

\section{Left Ventricular Pressure Overload and Extracellular Matrix Post-Translational Events}

This study assessed the effects of LVPO on myocardial collagen content and collagen stability. LVPO was associated with a significant increase in myocardial collagen content. Moreover, insoluble collagen content, indicative of collagen cross-linking and stability, was also increased with LVPO, and a relationship between myocardial collagen content and regional myocardial stiffness was demonstrated. Of note, these changes in collagen content and cross-linking occurred in the absence of demonstrable changes in mRNA

fibrillar collagen levels. Although these measurements were performed at one point in time, and only after the LV remodeling process with LVPO had become manifest, these findings do suggest that persistent collagen transcriptional activity is not the sole determinant of matrix accumulation. Important post-transcriptional/translational determinants of ECM remodeling include the MMPs and TIMPs. MMPs are a large family of proteolytic enzymes that include both soluble and membrane bound species. ${ }^{12}$ These enzymes are capable of degrading all components of the ECM, and their activity is regulated by TIMPs. ${ }^{12}$ MMP activity and TIMP abundance have been linked to myocardial fibrosis in LVPO. ${ }^{9,11,24-26}$ This study examined the change in mRNA levels for selected MMPs from various classes, including the gelatinases, matrilysins, collagenases, and membrane-type MMPs. Despite significant LV hypertrophy and ECM accumulation, no parallel change in the ubiquitous collagenase MMP-13 or the gelatinases MMP- 2 or 9 occurred. Other MMP types (eg, MMP-7) were actually reduced with LVPO. MMP-7 is an MMP type expressed in macrophages and fibroblasts. ${ }^{27}$ Although it remains speculative, the relative reduction in this MMP type suggests that a phenotypic change in these cell types occurred with LVPO. However, the current study did not quantify or isolate these critical cell types that likely contributed to changes in the structure and function of the ECM with LVPO. In contradistinction to the above observations, the membrane-type MMP, MT1-MMP, increased with LVPO. This MMP-type processes a number of biological signaling molecules, most notably the profibrotic moiety transforming growth factor. ${ }^{28}$ For example, in a recent study, myocardial overexpression of MT1-MMP did not result in ECM degradation, but rather enhanced myocardial fibrosis. ${ }^{28}$ Thus, the induction of this MT1-MMP type would be consistent with the fibrillar collagen accumulation observed in the present study with LVPO.

A crucial determinant of the myocardial ECM structure is the interaction between the MMPs and their endogenous tissue inhibitors, or TIMPs. ${ }^{28}$ TIMPs are small molecular weight proteins that can demonstrate a diversity of biologic functions $^{29,30}$ beyond that of MMP inhibition alone. For example, TIMPs can facilitate ECM accumulation not only by attenuating overall net MMP proteolytic activity but also by inducing profibrotic cascades in and of themselves. ${ }^{31}$ In the present study, LVPO caused a robust increase in TIMP-1 and TIMP-4 mRNA levels that paralleled fibrillar matrix accumulation. These particular TIMPs have been identified to regulate fibroblast growth, viability, and proliferation in vitro. ${ }^{31}$ Thus, the induction of these TIMPs with LVPO likely altered several cellular and biological processes relevant to fibrillar collagen accumulation from reducing MMP activity, inducing profibrotic pathways, and enhancing fibroblast function. Although remaining speculative, it is likely that the induction of these TIMPs may constitute an important event in the progression of ECM remodeling and LV diastolic dysfunction that occurs with LVPO. Indeed, clinical studies of LVPO have identified that increased plasma levels of TIMP-1 and 4 occur in patients with developing heart failure, secondary to diastolic dysfunction. ${ }^{17,24}$ In the present study, the diastolic dysfunction that occurred secondary to the induction of LVPO resulted in a significant increase in plasma levels of TIMP-1 and 4. The relative magnitude of the elevated plasma TIMP profiles observed in this large animal model of LVPO was similar to that observed previously in patients with LVPO and diastolic heart failure. ${ }^{18,24}$ Thus, future studies focusing on modulating relative TIMP-1 and 4 levels in this model of LVPO may hold both diagnostic and therapeutic relevance.

\section{CONCLUSIONS}

Progressive induction of LVPO in this large animal model related to LV function and stiffness elicited a unique matrix signature and was associated with myocardial ECM remodeling. Specifically, an increase in collagen content and stability and a reduction in the indices of collagen degradation were observed. These changes seemed to occur in the absence of increased collagen expression. Finally, although associative, the present study demonstrated a relationship between the induction of LVPO, myocyte growth, and ECM remodeling and the indices of diastolic dysfunction. Thus, this large animal model of LVPO will allow for mechanistic assessment of how specific cellular and extracellular pathways may contribute specifically to LV diastolic dysfunction in the absence of severe systolic failure, an important and clinically relevant scientific objective. ${ }^{32}$ However, it must also be recognized that no animal model can completely recapitulate the clinical presentation of chronic LVPO such as that with aortic stenosis. For example, the present study used sequential ascending supravalvular aortic constriction. This model of LVPO would unlikely cause the changes in coronary blood flow and therefore the ischemic substrate that would be operative 
in patients with valvular aortic stenosis. Second, the stepped and sequential LVPO induced in the present study is unlikely to completely represent the duration, pattern, and magnitude that occur in clinical forms of LVPO. Nevertheless, this model of LVPO demonstrated significant phenotypic similarities to clinical observations of LVPO in patients in terms of myocardial ECM remodeling and LV diastolic dysfunction. The model system and paradigms identified in this article may provide a means to identify the mechanisms that give rise to pathologic ECM remodeling and the potential point of irreversible ECM remodeling, and thereby improve the timing and treatment approaches for elimination of LVPO stimulus.

\section{References}

1. Monrad ES, Hess OM, Murakami T, et al. Time course of regression of left ventricular hypertrophy after aortic valve replacement. Circulation. 1988;77: 1345-55.

2. Villari B, Vassalli G, Monrad ES, et al. Normalization of diastolic dysfunction in aortic stenosis late after valve replacement. Circulation. 1995;91:2353-8.

3. Krayenbuehl HP, Hess OM, Monrad ES, et al. Left ventricular myocardial structure in aortic valve disease before, intermediate, and late after aortic valve replacement. Circulation. 1989;79:744-55.

4. Hess OM, Ritter M, Schneider J, et al. Diastolic stiffness and myocardial structure in aortic valve disease before and after valve replacement. Circulation. 1984; 69:855-65.

5. Azevedo CF, Nigri M, Higuchi ML, et al. Prognostic significance of myocardial fibrosis quantification by histopathology and magnetic resonance imaging in patients with severe aortic valve disease. J Am Coll Cardiol. 2010;56: 278-87.

6. Villar AV, Llano M, Cobo M, et al. Gender differences of echocardiographic and gene expression patterns in human pressure overload left ventricular hypertrophy. J Mol Cell Cardiol. 2009;46:526-35.

7. Kuwahara F, Kai H, Tokuda K, et al. Transforming growth factor-beta function blocking prevents myocardial fibrosis and diastolic dysfunction in pressureoverloaded rats. Circulation. 2002;106:130-5.

8. Moorjani N, Ahmad M, Catarino P, et al. Activation of apoptotic caspase cascade during the transition to pressure overload-induced heart failure. J Am Coll Cardiol. 2006;48:1451-8.

9. Schubert A, Walther T, Falk V, et al. Extracellular matrix gene expression correlates to left ventricular mass index after surgical induction of left ventricular hypertrophy. Basic Res Cardiol. 2001;96:381-7.

10. Nagatomo Y, Carabello BA, Coker ML, et al. Differential effects of pressure or volume overload on myocardial MMP levels and inhibitory control. Am J Physiol Heart Circ Physiol. 2000;278:H151-61.

11. Walther T, Schubert A, Falk V, et al. Regression of left ventricular hypertrophy after surgical therapy for aortic stenosis is associated with changes in extracellular matrix gene expression. Circulation. 2001;104(Suppl I):I-54-8.

12. Deardorff R, Spinale FG. Cytokines and matrix metalloproteinases as potential biomarkers in chronic heart failure. Biomark Med. 2009;3:513-23.

13. Deschamps AM, Spinale FG. Pathways of matrix metalloproteinase induction in heart failure: bioactive molecules and transcriptional regulation. Cardiovasc Res. 2006;69:666-76.

14. Nimmi ME. Fibrillar collagens: their biosynthesis, molecular structure, and mode of assembly. In: Zern MA, Reid LM, eds. Extracellular Matrix. New York: Marcel Decker; 1993:121-48.

15. López B, González A, Hermida N, et al. Role of lysyl oxidase in myocardial fibrosis: from basic science to clinical aspects. Am J Physiol Heart Circ Physiol. 2010;299:H1-9.

16. Yarbrough WM, Mukherjee R, Brinsa TA, et al. Matrix metalloproteinase inhibition modifies left ventricular remodeling after myocardial infarction in pigs. J Thorac Cardiovasc Surg. 2003;125:602-10.

17. Zile MR, DeSantis SM, Baicu CF, et al. Plasma biomarkers that reflect determinants of matrix composition identify the presence of left ventricular hypertrophy and diastolic heart failure. Circ Heart Fail. 2011;4:246-56.

18. Spinale FG, Coker ML, Thomas CV, et al. Time-dependent changes in matrix metalloproteinase activity and expression during the progression of congestive heart failure: relation to ventricular and myocyte function. Circ Res. 1998;82: 482-95.

19. Lindsey ML, Goshorn DK, Squires CE, et al. Age-dependent changes in myocardial matrix metalloproteinase/tissue inhibitor of metalloproteinase profiles and fibroblast function. Cardiovasc Res. 2005;66:410-9.

20. Marotta M, Martino G. Sensitive spectrophotometric method for the quantitative estimation of collagen. Anal Biochem. 1985;150:86-90.

21. Walsh BJ, Thornton SC, Penny R, Breit SN. Microplate reader-based quantitation of collagens. Anal Biochem. 1992;203:187-90.

22. Dahl JS, Videbaek L, Poulsen MK, et al. Effect of candesartan treatment on left ventricular remodeling after aortic valve replacement for aortic stenosis. Am J Cardiol. 2010;106:713-9.

23. Monrad ES, Hess OM, Murakami T, et al. Abnormal exercise hemodynamics in patients with normal systolic function late after aortic valve replacement. Circulation. 1988;77:613-24.

24. Ahmed SH, Clark LL, Pennington WR, et al. Matrix metalloproteinases/tissue inhibitors of metalloproteinases: relationship between changes in proteolytic determinants of matrix composition and structural, functional, and clinical manifestations of hypertensive heart disease. Circulation. 2006;113:2089-96.

25. Heymans S, Schroen B, Vermeersch P, et al. Increased cardiac expression of tissue inhibitor of metalloproteinase- 1 and tissue inhibitor of metalloproteinase- 2 is related to cardiac fibrosis and dysfunction in the chronic pressure-overloaded human heart. Circulation. 2005;112:1136-44.

26. Foronjy RF, Sun J, Lemaitre V, et al. Transgenic expression of matrix metalloproteinase-1 inhibits myocardial fibrosis and prevents the transition to heart failure in a pressure overload mouse model. Hypertens Res. 2008;31: 725-35.

27. Burke B. The role of matrix metalloproteinase 7 in innate immunity. Immunobiology. 2004;209:51-6.

28. Spinale FG, Mukherjee R, Zavadzkas JA, et al. Cardiac restricted overexpression of membrane type-1 matrix metalloproteinase causes adverse myocardial remodeling following myocardial infarction. J Biol Chem. 2010;285:30316-27.

29. Tummalapalli CM, Heath BJ, Tyagi SC. Tissue inhibitor of metalloproteinase-4 instigates apoptosis in transformed cardiac fibroblasts. J Cell Biochem. 2001;80: 512-21.

30. Stetler-Stevenson WG. The tumor microenvironment: regulation by MMPindependent effects of tissue inhibitor of metalloproteinases-2. Cancer Metastasis Rev. 2008;27:57-66.

31. Lovelock JD, Baker AH, Gao F, et al. Heterogeneous effects of tissue inhibitors of matrix metalloproteinases on cardiac fibroblasts. Am J Physiol Heart Circ Physiol. 2005;288:H461-8.

32. Yarbrough WM, Mukherjee R, Ikonomidis JS, Zile MR, Spinale FG. Myocardial remodeling with aortic stenosis and after aortic valve replacement: mechanisms and future prognostic implications. J Thorac Cardiovasc Surg. 2011 Jul 13 [Epub ahead of print].

\section{Discussion}

Dr Joseph Schmoker (Burlington, Vt). I have no disclosures. My questions pertain to the miR-1 and 133 that you studied. In the mouse model, the transverse aortic constriction (TAC) model, both are actually antihypertrophic and overexpression of miR-133 is associated with down-regulation of collagen in cultured fibroblasts. So I'm curious how you interpret this relative to the opposite findings in your large animal model. In addition, did you perform microarray analysis on myocardium to broaden your miR phenotype?

Dr Yarbrough. We have just begun our initial assessments with respect to miRs. We anticipate performing microarray analysis to broaden the miR phenotype. I agree these initial preliminary miR data are at odds with the relatively small amount of data that exist in the literature with respect to this topic.

At this time, we believe that miR-133 in particular may inhibit collagen $1 \mathrm{~A} 1$ and $3 \mathrm{~A} 1$. Our theory is that with up-regulation of miR-133, there is inhibition of collagen $1 \mathrm{~A} 1$ and $3 \mathrm{~A} 1$ expression. That would account for the lack of increased collagen expression 
noted in the study. In summary, we believe the increased collagen content observed in the study was secondary to increased collagen stability and resistance to degradation as opposed to increased collagen expression. With prolongation of the animal model, we may see a reduction in miR-133 and miR-1 levels that will then "release" some of the inhibition on the collagen $1 \mathrm{~A} 1$ and $3 \mathrm{~A} 1$ genes. That may then lead to a robust increase in collagen expression, counter to what we're seeing at this point in time at 1 month.

Dr Schmoker. My final question is related to how the control animals were handled at the terminal data collection. My understanding from the article is that the controls were not violated from the standpoint of manipulation of the heart during final data collection. Knowing that both miR-1 and 133 can be induced with myocardial ischemia, could subendocardial ischemia incurred from surgical handling and manipulation during terminal data collection be responsible for the differences in miR expression in the experimental group relative to controls?

Dr Yarbrough. Perhaps, but I don't believe that is likely. We performed microsphere studies on both the control and the instrumented animals and showed no difference in perfusion with respect to microsphere data collected from the endocardium, myocardium, or epicardium. Thus, I don't think submyocardial ischemia was likely. 